\title{
Branching fractions and $\boldsymbol{A}$ values in singly ionized tantalum (Ta II)
}

\author{
P. Quinet ${ }^{1,2}$, V. Fivet ${ }^{1}$, P. Palmeri ${ }^{1}$, É. Biémont ${ }^{1,2}$, L. Engström ${ }^{3}$, H. Lundberg ${ }^{3}$, and H. Nilsson ${ }^{4}$ \\ 1 Astrophysique et Spectroscopie, Université de Mons-Hainaut, 7000 Mons, Belgium \\ e-mail: E. Biemont@ulg.ac. be \\ 2 IPNAS, Université de Liège, Sart Tilman, 4000 Liège, Belgium \\ 3 Department of Physics, Lund Institute of Technology, PO Box 118, 22100 Lund, Sweden \\ ${ }^{4}$ Lund Observatory, Lund University, PO Box 43, 22100 Lund, Sweden
}

Received 25 September 2008 / Accepted 23 October 2008

\section{ABSTRACT}

\begin{abstract}
Aims. We report on the theoretical and experimental lifetimes of Ta II, and calculated branching fractions for selected transitions. Methods. The theoretical data are obtained by means of a relativistic Hartree-Fock method with detailed attention to correlation effects. The experimental lifetimes are measured with the time-resolved, laser-induced, fluorescence technique. Results. The calculated results are in good agreement with both previously known and new experimental lifetimes. New transition probabilities have been deduced for a set of Ta II transitions of astrophysical interest.
\end{abstract}

Key words. atomic processes - line: identification

\section{Introduction}

There is growing interest in atomic data of heavy ions in different fields of physics including plasma diagnostics in fusion research, absorption spectroscopy in environmental studies, and investigations of the chemical composition of astrophysical objects. As part of an on-going effort to meet this demand, we report on new transition probabilities in singly ionized tantalum (Ta II).

The richness and complexity of the Ta II spectrum impose severe limitations in determining absolute transition probabilities from either lifetime measurements or by absorption and emission methods, because of the need for reliable branching fractions. The experimental determination of these quantities is affected by: fragmentary knowledge of the atomic structure of this ion, even for low-lying configurations; intensity calibration over the wide wavelength range that must be considered and possible "missing" weak branches. As a consequence, the experimental transition probabilities are rather scarse in heavy ions in general, and in Ta II in particular, and must be supplemented by theoretical results.

The singly charged ion $\mathrm{Ta}^{+}$has strong $5 \mathrm{~d}^{3} 6 \mathrm{~s}-5 \mathrm{~d}^{3} 6 \mathrm{p}$ transitions between levels of high $J$ arising within terms of maximum spin. These transitions are suitable for diagnostic purposes when interpreting astrophysical spectra. However, they are strongly influenced by screening effects, configuration interaction, and relativistic contributions. They appear as an interesting challenge for atomic physicists because the theoretical lifetimes and branching fractions are very sensitive to the details of the configuration interaction included in the calculations.

In astrophysics, investigations of the tantalum abundance in the stars have been scarce partly due to the lack of strong transitions observable in accessible spectral ranges. So far, the solar photospheric abundance of tantalum has not yet been determined (see e.g. Asplund et al. 2006). However, Ta II has been investigated in the spectra of chemically peculiar stars including $\chi$ Lupi (see e.g. Eriksson et al. 2002).
We present a detailed set of calculated transition probabilities for many transitions in Ta II. The only data available of this nature are those of Corliss \& Bozman (1962), which are well known to be affected by large systematic errors. The accuracy of the present results is assessed by comparison between theoretical and experimental lifetimes obtained here and in previous investigations.

The present work extends some results reported in neutral (Fivet et al. 2006) and also in doubly ionized tantalum (Ta III) (Fivet et al. 2008).

\section{The theoretical model}

According to Moore (1958), the ground state of Ta II is $5 d^{3}\left({ }^{4} F\right) 6 s^{5} F_{1}$, and the first excited configurations are $5 d^{2} 6 s^{2}$ and $5 \mathrm{~d}^{4}$, which are of the same parity (even) as the ground configuration. Extensive contributions to the analysis of the Ta II spectrum are due to Kiess (1962) and, later on, to Wyart (1977) and Wyart \& Blaise (1990). The latter investigations led to the elimination of "fortuitous" levels reported in earlier analyses. Our present investigation is based on these three analyses of Ta II.

Configuration interactions appear strong in between the $5 \mathrm{~d}^{N} 6 \mathrm{p}, 5 \mathrm{~d}^{N-1} 6 \mathrm{~s} 6 \mathrm{p}$, and $5 \mathrm{~d}^{N-2} 6 \mathrm{~s}^{2} 6 \mathrm{p}$ odd-parity configurations in the platinum-group elements, the latter configuration being generally unrecognizable. For the even-parity states, the strong interactions between $5 \mathrm{~d}^{N}, 5 \mathrm{~d}^{N-1} 6 \mathrm{~s}$, and $5 \mathrm{~d}^{N-2} 6 \mathrm{~s}^{2}$ configurations are well known and have been investigated in a systematic way with the parametric method (Wyart 1977).

Atomic-structure calculations in a heavy ion such as Ta II are realistic only if relativistic and correlation effects are considered simultaneously in the physical model. In the past, we performed a large number of atomic-structure calculations (see e.g. Biémont \& Quinet 2003; Biémont 2005) using HFR approach (Cowan 1981), which is based on the non-relativistic Schrödinger equation but includes the most significant relativistic effects (Blume-Watson spin-orbit interaction, mass-velocity, 
Table 1. Configurations considered for the two models retained for Ta II. For more details, see text.

\begin{tabular}{llll}
\hline \hline HFR + CP(A) & & HFR + CP(B) \\
Even config. & Odd config. & Even config. & Odd config. \\
\hline $5 \mathrm{~d}^{3} 6 \mathrm{~s}$ & $5 \mathrm{~d}^{3} 6 \mathrm{p}$ & $5 \mathrm{~d}^{3} 6 \mathrm{~s}$ & $5 \mathrm{~d}^{3} 6 \mathrm{p}$ \\
$5 \mathrm{~d}^{3} 7 \mathrm{~s}$ & $5 \mathrm{~d}^{3} 7 \mathrm{p}$ & $5 \mathrm{~d}^{3} 7 \mathrm{~s}$ & $5 \mathrm{~d}^{3} 7 \mathrm{p}$ \\
$5 \mathrm{~d}^{3} 6 \mathrm{~d}$ & $5 \mathrm{~d}^{3} 5 \mathrm{f}$ & $5 \mathrm{~d}^{3} 6 \mathrm{~d}$ & $5 \mathrm{~d}^{3} 5 \mathrm{f}$ \\
$5 \mathrm{~d}^{4}$ & $5 \mathrm{~d}^{3} 6 \mathrm{f}$ & $5 \mathrm{~d}^{4}$ & $5 \mathrm{~d}^{3} 6 \mathrm{f}$ \\
$5 \mathrm{~d}^{2} 6 \mathrm{~s}^{2}$ & $5 \mathrm{~d}^{2} 6 \mathrm{~s} 6 \mathrm{p}$ & $5 \mathrm{~d}^{2} 6 \mathrm{~s}^{2}$ & $5 \mathrm{~d}^{2} 6 \mathrm{~s} 6 \mathrm{p}$ \\
$5 \mathrm{~d}^{2} 6 \mathrm{p}^{2}$ & $5 \mathrm{~d}^{2} 6 \mathrm{~s} 7 \mathrm{p}$ & $5 \mathrm{~d}^{2} 6 \mathrm{p}^{2}$ & $5 \mathrm{~d}^{2} 6 \mathrm{~s} 7 \mathrm{p}$ \\
$5 \mathrm{~d}^{2} 6 \mathrm{~d}^{2}$ & $5 \mathrm{~d}^{2} 6 \mathrm{~s} 5 \mathrm{f}$ & $5 \mathrm{~d}^{2} 6 \mathrm{~d}^{2}$ & $5 \mathrm{~d}^{2} 6 \mathrm{~s} 5 \mathrm{f}$ \\
$5 \mathrm{~d}^{2} 7 \mathrm{~s}^{2}$ & $5 \mathrm{~d}^{2} 6 \mathrm{~s} 6 \mathrm{f}$ & $5 \mathrm{~d}^{2} 7 \mathrm{~s}^{2}$ & $5 \mathrm{~d}^{2} 6 \mathrm{~s} 6 \mathrm{f}$ \\
$5 \mathrm{~d}^{2} 6 \mathrm{~s} 6 \mathrm{~d}$ & $5 \mathrm{~d}^{2} 6 \mathrm{p} 6 \mathrm{~d}$ & $5 \mathrm{~d}^{2} 6 \mathrm{~s} 6 \mathrm{~d}$ & $5 \mathrm{~d}^{2} 6 \mathrm{p} 6 \mathrm{~d}$ \\
$5 \mathrm{~d}^{2} 6 \mathrm{~s} 7 \mathrm{~s}$ & $5 \mathrm{~d} 6 \mathrm{~s}^{2} 6 \mathrm{p}$ & $5 \mathrm{~d}^{2} 6 \mathrm{~s} 7 \mathrm{~s}$ & $5 \mathrm{~d}^{2} 6 \mathrm{~s}^{2} 6 \mathrm{p}$ \\
$5 \mathrm{~d} 6 \mathrm{~s}^{2} 6 \mathrm{~d}$ & $5 \mathrm{~d}^{2} 5 \mathrm{f}$ & $5 \mathrm{~d}^{2} 6 \mathrm{p} 5 \mathrm{f}$ & $5 \mathrm{~d}^{2} 6 \mathrm{p} 7 \mathrm{~s}$ \\
$5 \mathrm{~d} 6 \mathrm{~s}^{2} 7 \mathrm{~s}$ & $5 \mathrm{~d} 6 \mathrm{~s}^{2} 6 \mathrm{f}$ & $5 \mathrm{~d}^{2} 6 \mathrm{~d} 7 \mathrm{~s}$ & \\
$5 \mathrm{~d} 6 \mathrm{~s} 6 \mathrm{p}^{2}$ & $5 \mathrm{~d} 6 \mathrm{p}^{3}$ & & \\
$6 \mathrm{~s}^{2} 6 \mathrm{p}^{2}$ & $6 \mathrm{~s} 6 \mathrm{p}^{3}$ & & \\
$5 \mathrm{~d}^{2} 6 \mathrm{p} 5 \mathrm{f}$ & $5 \mathrm{~d}^{2} 6 \mathrm{p} 7 \mathrm{~s}$ & & \\
$6 \mathrm{~s}^{2} 6 \mathrm{p} 5 \mathrm{f}$ & $6 \mathrm{~s}^{2} 6 \mathrm{p} 7 \mathrm{~s}$ & & \\
$5 \mathrm{~d} 6 \mathrm{~s} 6 \mathrm{p} 5 \mathrm{f}$ & $5 \mathrm{~d} 6 \mathrm{~s} 6 \mathrm{p} 6 \mathrm{~d}$ & & \\
$5 \mathrm{~d}^{2} 6 \mathrm{~d} 7 \mathrm{~s}$ & $5 \mathrm{~d} 6 \mathrm{~s} 6 \mathrm{p} 7 \mathrm{~s}$ & & \\
$5 \mathrm{~d} 6 \mathrm{~s} 7 \mathrm{~s}^{2}$ & $6 \mathrm{~s}^{2} 6 \mathrm{p} 6 \mathrm{~d}$ & & \\
\hline
\end{tabular}

and one-body Darwin terms). Configuration interaction can be included in calculations in an extensive way using either an $\mathrm{ab}$ initio approach or a semi-empirical one based on a leastsquares fitting procedure applied to the calculated eigenvalues to obtain the most appropriate fit with the experimental energy levels. For more detailed and relevant references, we refer the reader to Biémont \& Quinet (2003) and Biémont (2005).

In the present calculation, valence-valence type interactions have been considered by including in the configurationinteraction expansions, the configurations presented in Table 1.

Core-valence interactions were taken into account by using a polarization-model potential and a correction to the dipole operator following a well-established procedure (see e.g. Quinet et al. 1999) giving rise to the so-called HFR + CP method. In the present context, two different polarization models were considered. In the first model $[\mathrm{HFR}+\mathrm{CP}(\mathrm{A})]$, we retained a $5 s^{2} 5 p^{6} 4 \mathrm{f}^{14} \mathrm{Ta}^{5+}$ ionic core surrounded by 4 valence electrons. For the dipole polarizability, we adopted the value $\alpha_{\mathrm{d}}=$ 3.18 a.u. (Fraga et al. 1976). The cut-off radius used was the HFR mean radius of the outermost core orbital and was chosen to equal $r_{\mathrm{c}}(5 \mathrm{p})=1.30$ a.u. In a second model $[\mathrm{HFR}+\mathrm{CP}(\mathrm{B})]$, a $\mathrm{Ta}^{3+}$ ionic core $\left(5 \mathrm{~s}^{2} 5 \mathrm{p}^{6} 4 \mathrm{f}^{14} 5 \mathrm{~d}^{2}\right)$ was considered to be surrounded by 2 valence electrons. The corresponding value of dipole polarisability was $\alpha_{\mathrm{d}}=6.75$ a.u., and the cut-off radius $r_{\mathrm{c}}(5 \mathrm{~d})$ equalled 1.95 a.u. The two sets of configurations adopted in the calculations are reported in Table 1.

In the fitting procedure, we used all the experimentally established levels from Kiess (1962), Wyart (1977), and Wyart \& Blaise (1990). All parameters related to the experimentally observed configurations have been adjusted to reproduce the experimental levels in the most accurate way. The standard deviations in the fitting procedures were $60 \mathrm{~cm}^{-1}$ and $120 \mathrm{~cm}^{-1}$ (model A), and $60 \mathrm{~cm}^{-1}$ and $140 \mathrm{~cm}^{-1}$ (model B), for the even and odd parities, respectively.

Comparisons between theoretical and experimental energy levels are reported in Tables 2 and 3 for the even and odd parities, respectively, and are limited to the levels $E<45000 \mathrm{~cm}^{-1}$ and $E<65000 \mathrm{~cm}^{-1}$. In the same tables, we also compare the experimental and calculated Landé $g$-factors obtained with the two different models. The two sets of $g$-values agree quite well and are also in excellent agreement with the available experimental results (Wyart 1977; Wyart \& Blaise 1990).

The calculated BFs for transitions originating in selected levels of Ta II are reported in Table 4, where contributions above 1\% only are quoted. Due to space limitations, the table is restricted to odd-parity levels with energy $E<44000 \mathrm{~cm}^{-1}$, for which experimental lifetimes are available (see Sect. 3). They should thus contain all the low-excitation transitions of astrophysical interest.

\section{The experimental lifetimes}

\subsection{Previous work}

Ta II lifetimes have been the subject of a number of experimental investigations. Six lifetimes were measured by Kwiatkowski et al. (1984) using selective laser excitation and time-resolved observation of the fluorescence light. The ions were produced by a sputtering technique in a low-pressure discharge. Pulsed laser excitation and time-resolved detection were applied by Bergström et al. (1986) in measuring 8 lifetimes using a lowpressure, hollow-cathode discharge as an ion source. Lifetimes of 10 levels in Ta II were obtained by Schade \& Helbig (1986) using a similar technique. This work was extended with the same approach to 15 additional levels by Langhans et al. (1995). The latest lifetime measurements in Ta II (6 levels) were performed by Henderson et al. (1999) with the beam-foil spectroscopy technique.

\subsection{New measurements}

We report on lifetime measurements of three short-lived, oddparity levels of Ta II obtained using TR-LIF spectroscopy on a laser-produced plasma. The TR-LIF technique has previously provided accurate lifetimes for neutral (Fivet et al. 2006) and doubly ionized tantalum (Fivet et al. 2008). Details of the experimental setup used in the present experiment were described elsewhere (see e.g. Bergström et al. 1986; Xu et al. 2003, 2004; Fivet et al. 2006, 2008) and only a brief description will be provided here.

Tantalum ions, in the ground as well as in excited states, were generated in a laser-produced plasma by focusing a $532 \mathrm{~nm}$ $\mathrm{Nd}$ :YAG laser pulse (Continuum Surelite) onto a rotating, tantalum target. The density and temperature of the expanding plasma were controlled and adjusted by changing the pulse energy and beam size incident on the target. The different ionization stages, having different velocities, could be separated by selecting an appropriate delay time between the ablation and excitation pulses.

The excitation pulses, obtained from a frequency-doubled Nd:YAG laser pulse (Continuum NY-82) transmitted into a temporal compressor, have a duration of 1-2 ns. To generate the required excitation wavelengths, the compressed pulses were used to pump a dye laser (Continuum Nd-60). Using the DCM dye, excitation wavelengths in the range 191-211 nm were obtained by non-linear processes, such as frequency doubling and tripling using KDP and BBO crystals and stimulated Raman scattering in a hydrogen-gas cell.

The excitation beam interacted with the tantalum ions about $1 \mathrm{~cm}$ above the target. The fluorescence, emitted from the excited levels, was collected by a fused-silica lens and focused onto the entrance slit of a $1 / 8 \mathrm{~m}$ monochromator, and then detected by a Hamamatsu 1564U micro-channel-plate photomultiplier tube 
Table 2. Experimental and calculated energies (in $\left.\mathrm{cm}^{-1}\right)$ and Landé $g$-factors for the lowest even-parity levels $\left(E<45000 \mathrm{~cm}^{-1}\right)$ of Ta II.

\begin{tabular}{|c|c|c|c|c|c|c|c|c|}
\hline$J$ & $E(\mathrm{EXP})^{a}$ & $g(\mathrm{EXP})^{a}$ & $E(\mathrm{CALC})^{b}$ & $g(\mathrm{CALC})^{b}$ & $\Delta E^{b}$ & $E(\mathrm{CALC})^{c}$ & $g(\mathrm{CALC})^{c}$ & $\Delta E^{c}$ \\
\hline 1 & 0.00 & 0.000 & -27 & 0.035 & 27 & -32 & 0.035 & 32 \\
\hline 2 & 1031.36 & 1.008 & 1023 & 1.008 & 8 & 1021 & 1.009 & 10 \\
\hline 3 & 2642.26 & 1.250 & 2641 & 1.247 & 1 & 2642 & 1.247 & 0 \\
\hline 2 & 3180.04 & 0.750 & 3209 & 0.757 & -29 & 3218 & 0.755 & -38 \\
\hline 0 & 4124.85 & & 4074 & & 51 & 4062 & & 63 \\
\hline 4 & 4415.79 & 1.350 & 4410 & 1.339 & 6 & 4412 & 1.339 & 4 \\
\hline 1 & 5330.77 & 1.550 & 5303 & 1.559 & 28 & 5296 & 1.559 & 35 \\
\hline 2 & 5657.90 & 1.340 & 5645 & 1.329 & 13 & 5651 & 1.332 & 7 \\
\hline 5 & 6186.81 & 1.410 & 6175 & 1.381 & 12 & 6175 & 1.381 & 12 \\
\hline 3 & 6831.31 & 1.098 & 6858 & 1.083 & -27 & 6852 & 1.083 & -21 \\
\hline 2 & 9690.47 & 1.047 & 9678 & 1.066 & 12 & 9679 & 1.224 & 11 \\
\hline 4 & 9746.28 & 1.225 & 9688 & 1.223 & 58 & 9698 & 1.065 & 48 \\
\hline 1 & 10713.21 & 2.353 & 10778 & 2.361 & -65 & 10781 & 2.361 & -68 \\
\hline 3 & 11767.16 & 0.908 & 11740 & 0.919 & 27 & 11733 & 0.916 & 34 \\
\hline 2 & 11875.47 & 1.426 & 12003 & 1.399 & -128 & 12010 & 1.402 & -135 \\
\hline 3 & 12435.85 & 1.594 & 12406 & 1.606 & 30 & 12410 & 1.607 & 26 \\
\hline 0 & 12600.87 & & 12589 & & 12 & 12591 & & 10 \\
\hline 4 & 12705.33 & 1.019 & 12626 & 1.032 & 79 & 12627 & 1.032 & 78 \\
\hline 4 & 12966.02 & 1.040 & 13073 & 1.039 & -107 & 13072 & 1.038 & -106 \\
\hline 1 & 13475.38 & 1.498 & 13494 & 1.515 & -19 & 13492 & 1.514 & -17 \\
\hline 2 & 13560.24 & 1.120 & 13593 & 1.135 & -33 & 13609 & 1.133 & -49 \\
\hline 5 & 14158.51 & 1.150 & 14261 & 1.154 & -102 & 14245 & 1.154 & -86 \\
\hline 2 & 14494.87 & 1.476 & 14501 & 1.469 & -6 & 14497 & 1.006 & -2 \\
\hline 3 & 14581.07 & 0.988 & 14488 & 1.004 & 93 & 14496 & 1.469 & 85 \\
\hline 1 & 14627.64 & 0.854 & 14638 & 0.841 & -10 & 14623 & 0.842 & 5 \\
\hline 3 & 15726.15 & 1.455 & 15720 & 1.461 & 6 & 15716 & 1.461 & 10 \\
\hline 4 & 15851.12 & 1.140 & 15747 & 1.150 & 104 & 15752 & 1.149 & 99 \\
\hline 0 & 16288.04 & & 16378 & & -90 & 16364 & & -76 \\
\hline 2 & 17168.48 & 1.204 & 17143 & 1.222 & 25 & 17138 & 1.221 & 30 \\
\hline 4 & 17231.19 & 1.191 & 17206 & 1.151 & 25 & 17197 & 1.152 & 34 \\
\hline 1 & 17375.00 & 1.170 & 17420 & 1.187 & -45 & 17402 & 1.187 & -27 \\
\hline 6 & 17982.00 & 1.146 & 18054 & 1.167 & -72 & 18038 & 1.167 & -56 \\
\hline 5 & 18186.04 & 1.100 & 18162 & 1.091 & 24 & 18149 & 1.091 & 37 \\
\hline 4 & 18493.66 & 1.227 & 18448 & 1.246 & 46 & 18433 & 1.439 & 61 \\
\hline 2 & 18500.62 & 1.441 & 18454 & 1.439 & 47 & 18450 & 1.247 & 51 \\
\hline 3 & 18553.83 & 1.355 & 18514 & 1.333 & 40 & 18504 & 1.333 & 50 \\
\hline 2 & 22928.61 & 0.700 & 22977 & 0.705 & -48 & 23011 & 0.711 & -82 \\
\hline 4 & 23082.71 & 1.026 & 23038 & 1.023 & 45 & 23048 & 1.020 & 35 \\
\hline 2 & 23294.77 & 1.118 & 23232 & 1.106 & 63 & 23202 & 1.099 & 93 \\
\hline 0 & 23381.28 & & 23304 & & 77 & 23302 & & 79 \\
\hline 1 & 23406.13 & & 23445 & 1.163 & -39 & 23477 & 1.165 & -71 \\
\hline 3 & 23620.35 & 1.076 & 23658 & 1.073 & -38 & 23692 & 1.072 & -72 \\
\hline 5 & 24226.20 & 1.000 & 24233 & 1.009 & -7 & 24262 & 1.009 & -36 \\
\hline 4 & 24432.83 & 0.985 & 24581 & 0.998 & -148 & 24573 & 0.991 & -140 \\
\hline 3 & 24869.58 & 0.995 & 24875 & 0.985 & -5 & 24882 & 0.986 & -12 \\
\hline 4 & 25385.49 & 1.085 & 25315 & 1.094 & 70 & 25305 & 1.103 & 80 \\
\hline 5 & 25414.13 & 1.060 & 25457 & 1.056 & -43 & 25455table & 1.056 & -41 \\
\hline 6 & 26010.70 & 1.117 & 26043 & 1.136 & -32 & 26038 & 1.136 & -27 \\
\hline 1 & 26234.60 & 1.332 & 26298 & 1.342 & -63 & 26302 & 1.340 & -67 \\
\hline 0 & 26722.38 & & 26654 & & 68 & 26647 & & 75 \\
\hline 3 & 26829.13 & 0.850 & 26820 & 0.851 & 9 & 26827 & 0.850 & 2 \\
\hline 2 & 28044.14 & 1.358 & 28048 & 1.367 & -4 & 28053 & 1.368 & -9 \\
\hline 4 & 28165.40 & 1.094 & 28171 & 1.055 & -6 & 28176 & 1.056 & -11 \\
\hline 2 & 29843.58 & 0.833 & 29803 & 0.823 & 41 & 29810 & 0.818 & 34 \\
\hline 1 & 29963.20 & 1.004 & 29987 & 1.020 & -24 & 29983 & 1.034 & -20 \\
\hline 5 & 30349.83 & 1.165 & 30289 & 1.177 & 61 & 30305 & 1.177 & 45 \\
\hline 2 & 30405.61 & 1.051 & 30395 & 1.083 & 11 & 30405 & 1.088 & 1 \\
\hline 3 & 30624.09 & 1.257 & 30665 & 1.247 & -41 & 30676 & 1.246 & -52 \\
\hline 6 & 30954.00 & 1.020 & 30963 & 1.031 & -9 & 30955 & 1.031 & -1 \\
\hline 4 & 31267.10 & 1.120 & 31204 & 1.126 & 63 & 31183 & 1.125 & 84 \\
\hline 3 & 31531.70 & 1.130 & 31516 & 1.125 & 16 & 31533 & 1.126 & -1 \\
\hline 0 & 31924.75 & & 31947 & & -22 & 31948 & & -23 \\
\hline 1 & 31948.68 & 1.000 & 31979 & 0.981 & -30 & 31989 & 0.966 & -40 \\
\hline 2 & 33027.18 & 1.020 & 33203 & 1.072 & -176 & 33204 & 1.075 & -177 \\
\hline 2 & 34879.69 & 1.400 & 34973 & 1.379 & -93 & 34973 & 1.375 & -93 \\
\hline 4 & 35151.35 & 1.078 & 35080 & 1.084 & 71 & 35087 & 1.085 & 64 \\
\hline
\end{tabular}


Table 2. continued.

\begin{tabular}{rrrrrrrrr}
\hline \hline$J$ & $E(\mathrm{EXP})^{a}$ & $g(\mathrm{EXP})^{a}$ & $E(\mathrm{CALC})^{b}$ & $g(\mathrm{CALC})^{b}$ & $\Delta E^{b}$ & $E(\mathrm{CALC})^{c}$ & $g(\mathrm{CALC})^{c}$ & $\Delta E^{c}$ \\
\hline 3 & 36614.88 & 1.235 & 36538 & 1.307 & 77 & 36544 & 1.307 & 71 \\
2 & 37274.31 & 1.105 & 37192 & 1.118 & 82 & 37203 & 1.119 & 71 \\
1 & & & 37379 & 0.508 & & 37415 & 0.508 & \\
0 & & & 37976 & & & 37922 & & \\
3 & 40369.15 & 1.050 & 40440 & 1.072 & -71 & 40437 & 1.072 & -68 \\
4 & 40904.37 & 1.220 & 40795 & 1.224 & 109 & 40786 & 1.224 & 118 \\
2 & & & 41120 & 0.709 & & 41123 & 0.709 & \\
2 & 41513.54 & 1.460 & 41571 & 1.479 & -57 & 41583 & 1.479 & -69 \\
3 & 43606.36 & & 43574 & 1.027 & 32 & 43579 & 1.027 & 27 \\
1 & & & 44136 & 1.493 & & 44159 & 1.493 & \\
\hline
\end{tabular}

${ }^{a}$ From Wyart (1977) and Wyart \& Blaise $(1990) ;{ }^{b}$ this work: model [HFR + CP(A)]; ${ }^{c}$ this work: model [HFR + CP(B)].

Table 3. Experimental and calculated energies (in $\left.\mathrm{cm}^{-1}\right)$ and Landé $g$-factors for the lowest odd-parity levels $\left(E<65000 \mathrm{~cm}^{-1}\right)$ of Ta II.

\begin{tabular}{|c|c|c|c|c|c|c|c|c|}
\hline$J$ & $E(\mathrm{EXP})^{a}$ & $g(\mathrm{EXP})^{a}$ & $E(\mathrm{CALC})^{b}$ & $g(\text { CALC })^{b}$ & $\Delta E^{b}$ & $E(\mathrm{CALC})^{c}$ & $g(\mathrm{CALC})^{c}$ & $\Delta E^{c}$ \\
\hline 2 & 29256.87 & 0.511 & 29523 & 0.499 & -266 & 29467 & 0.506 & -210 \\
\hline 3 & 32318.44 & 0.976 & 32338 & 0.975 & -20 & 32310 & 0.977 & 8 \\
\hline 1 & 33706.47 & 0.285 & 33487 & 0.231 & 219 & 33554 & 0.233 & 152 \\
\hline 2 & 33715.27 & 0.823 & 33850 & 0.890 & -135 & 33883 & 0.866 & -168 \\
\hline 4 & 36112.97 & 1.180 & 36083 & 1.168 & 30 & 36068 & 1.168 & 45 \\
\hline 2 & 36177.10 & 0.946 & 35993 & 0.859 & 184 & 36031 & 0.884 & 146 \\
\hline 3 & 36763.70 & 1.169 & 36874 & 1.200 & -110 & 36888 & 1.187 & -124 \\
\hline 1 & 36987.71 & 0.685 & 37208 & 0.753 & -220 & 37247 & 0.761 & -259 \\
\hline 0 & & & 37595 & & & 37660 & & \\
\hline 2 & 37230.75 & 0.623 & 37602 & 0.704 & -371 & 37553 & 0.700 & -322 \\
\hline 2 & 38515.55 & 1.006 & 38544 & 0.968 & -28 & 38532 & 0.986 & -16 \\
\hline 1 & 38535.21 & 0.472 & 38216 & 0.433 & 319 & 38315 & 0.471 & 220 \\
\hline 3 & 38962.32 & 0.976 & 39197 & 0.982 & -235 & 39025 & 1.139 & -63 \\
\hline 3 & 39295.81 & 1.138 & 39010 & 1.144 & 286 & 39164 & 0.992 & 132 \\
\hline 4 & 39743.67 & 1.223 & 39799 & 1.252 & -55 & 39767 & 1.244 & -23 \\
\hline 5 & 39758.81 & 1.270 & 39745 & 1.275 & 14 & 39723 & 1.274 & 36 \\
\hline 0 & 40023.68 & & 40023 & & 1 & 40121 & & -97 \\
\hline 2 & 40233.46 & 1.165 & 40263 & 1.108 & -30 & 40302 & 1.119 & -69 \\
\hline 1 & 40304.78 & 1.225 & 40210 & 1.177 & 95 & 40284 & 1.146 & 21 \\
\hline 2 & 41144.94 & 1.152 & 41025 & 1.165 & 120 & 41222 & 1.180 & -77 \\
\hline 1 & 41355.11 & 1.885 & 41508 & 1.911 & -153 & 41388 & 1.909 & -33 \\
\hline 3 & 41554.42 & 1.207 & 41507 & 1.242 & 47 & 41492 & 1.258 & 62 \\
\hline 5 & 41709.01 & 1.244 & 41759 & 1.257 & -50 & 41675 & 1.257 & 34 \\
\hline 4 & 41775.29 & 1.249 & 41815 & 1.244 & -40 & 41799 & 1.253 & -24 \\
\hline 4 & 42122.91 & 1.258 & 42034 & 1.268 & 89 & 42003 & 1.258 & 120 \\
\hline 2 & 42153.29 & 1.212 & 42086 & 1.202 & 67 & 42174 & 1.180 & -21 \\
\hline 3 & 42959.55 & 1.120 & 42926 & 1.127 & 34 & 42973 & 1.117 & -13 \\
\hline 2 & 43064.86 & 1.041 & 43022 & 1.089 & 43 & 43174 & 1.056 & -109 \\
\hline 0 & 43068.72 & & 43153 & & -84 & 43040 & & 29 \\
\hline 3 & 43544,46 & 1.059 & 43603 & 1.046 & -59 & 43598 & 1.035 & -54 \\
\hline 1 & 43553.67 & & 43547 & 1.420 & 7 & 43421 & 1.429 & 133 \\
\hline 4 & 44005.20 & 1.137 & 43949 & 1.208 & 56 & 43888 & 1.241 & 117 \\
\hline 1 & 44206.24 & 0.242 & 44004 & 0.313 & 202 & 44068 & 0.309 & 138 \\
\hline 2 & 44259.20 & 1.256 & 44140 & 1.253 & 119 & 44113 & 1.256 & 146 \\
\hline 3 & 44430.39 & 0.940 & 44442 & 0.954 & -12 & 44405 & 0.948 & 25 \\
\hline 0 & 44434.79 & & 44454 & & -19 & 44407 & & 28 \\
\hline 5 & 44585.17 & 1.297 & 44538 & 1.271 & 47 & 44549 & 1.274 & 36 \\
\hline 6 & & & 44580 & 1.331 & & 44480 & 1.331 & \\
\hline 4 & 44626.00 & 1.265 & 44660 & 1.184 & -34 & 44583 & 1.153 & 43 \\
\hline 3 & 44835.20 & 1.242 & 44747 & 1.229 & 88 & 44873 & 1.238 & -38 \\
\hline 1 & 45233.91 & 1.458 & 45229 & 1.446 & 5 & 45223 & 1.458 & 11 \\
\hline 2 & 45446.85 & 1.148 & 45470 & 1.098 & -23 & 45510 & 1.099 & -63 \\
\hline 1 & 46174.60 & 1.367 & 46121 & 1.359 & 54 & 46136 & 1.360 & 39 \\
\hline 5 & 46295.03 & 1.200 & 46472 & 1.202 & -177 & 46391 & 1.198 & -96 \\
\hline 2 & 46387.16 & 1.323 & 46509 & 1.337 & -122 & 46541 & 1.348 & -154 \\
\hline 4 & 46645.70 & 1.233 & 46487 & 1.216 & 159 & 46462 & 1.196 & 184 \\
\hline 3 & 46831.35 & 1.214 & 46714 & 1.198 & 117 & 46817 & 1.212 & 14 \\
\hline 2 & 46850.64 & 1.110 & 46910 & 1.016 & -59 & 47073 & 1.036 & -222 \\
\hline 3 & 47168.90 & 1.096 & 47128 & 1.097 & 41 & 47128 & 1.104 & 41 \\
\hline 4 & 47280.89 & 1.190 & 47268 & 1.223 & 13 & 47274 & 1.227 & 7 \\
\hline
\end{tabular}


Table 3. continued.

\begin{tabular}{|c|c|c|c|c|c|c|c|c|}
\hline$J$ & $E(\mathrm{EXP})^{a}$ & $g(\mathrm{EXP})^{a}$ & $E(\mathrm{CALC})^{b}$ & $g(\mathrm{CALC})^{b}$ & $\Delta E^{b}$ & $E(\mathrm{CALC})^{c}$ & $g(\mathrm{CALC})^{c}$ & $\Delta E^{c}$ \\
\hline 2 & 47514.52 & 1.284 & 47578 & 1.341 & -63 & 47609 & 1.304 & -94 \\
\hline 1 & 47595.98 & 1.520 & 47687 & 1.493 & -91 & 47767 & 1.519 & -171 \\
\hline 0 & 47801.03 & & 47853 & & -52 & 48003 & & -202 \\
\hline 3 & 47825.41 & 1.189 & 47775 & 1.190 & 50 & 47871 & 1.216 & -46 \\
\hline 6 & 47829.75 & 1.300 & 47751 & 1.299 & 79 & 47570 & 1.300 & 260 \\
\hline 0 & 48064.57 & & 48161 & & -96 & 48234 & & -169 \\
\hline 4 & 48162.10 & 1.030 & 48197 & 1.157 & -35 & 48183 & 1.057 & -21 \\
\hline 2 & 48223.08 & 1.446 & 48329 & 1.268 & -106 & 48279 & 1.262 & -56 \\
\hline 4 & 48470.41 & 1.276 & 48419 & 1.089 & 51 & 48468 & 1.203 & 2 \\
\hline 2 & 48666.56 & 1.131 & 48605 & 1.395 & 62 & 48598 & 1.519 & 69 \\
\hline 1 & 48776.29 & 0.746 & 48666 & 0.772 & 110 & 48818 & 0.830 & -42 \\
\hline 3 & 48962.54 & 1.283 & 48919 & 1.303 & 44 & 48926 & 1.342 & 37 \\
\hline 5 & 49055.18 & 1.212 & 48982 & 1.246 & 73 & 48915 & 1.248 & 140 \\
\hline 2 & 49080.51 & 1.320 & 49076 & 1.344 & 5 & 49083 & 1.255 & -2 \\
\hline 3 & 49536.24 & 1.317 & 49359 & 1.343 & 177 & 49363 & 1.280 & 173 \\
\hline 2 & 49592.90 & 1.629 & 49583 & 1.588 & 10 & 49365 & 1.613 & 228 \\
\hline 3 & 49646.62 & 1.048 & 49630 & 1.041 & 17 & 49685 & 1.053 & -38 \\
\hline 1 & 49886.97 & 2.006 & 49813 & 1.922 & 74 & 49653 & 2.066 & 234 \\
\hline 4 & 49937.74 & 1.207 & 49961 & 1.207 & -23 & 49911 & 1.219 & 27 \\
\hline 3 & 50314.43 & 1.281 & 50262 & 1.263 & 52 & 50201 & 1.268 & 113 \\
\hline 5 & 50507.12 & 1.080 & 50580 & 1.102 & -73 & 50604 & 1.103 & -97 \\
\hline 1 & 50531.17 & 1.370 & 50224 & 1.535 & 307 & 50134 & 1.416 & 397 \\
\hline 3 & 51073.92 & 1.156 & 51089 & 1.209 & -15 & 50907 & 1.247 & 167 \\
\hline 2 & 51197.42 & 1.359 & 51205 & 1.350 & -8 & 51187 & 1.356 & 10 \\
\hline 1 & 51326.31 & 1.047 & 51218 & 1.124 & 108 & 51287 & 1.444 & 39 \\
\hline 0 & 51334.37 & & 51285 & & 49 & 51043 & & 291 \\
\hline 4 & 51479.86 & 1.095 & 51454 & 1.124 & 26 & 51537 & 1.127 & -57 \\
\hline 2 & 51534.28 & 1.299 & 51693 & 1.285 & -159 & 51729 & 1.277 & -195 \\
\hline 5 & 51753.70 & 1.102 & 51916 & 1.105 & -162 & 51809 & 1.107 & -55 \\
\hline 3 & 52121.15 & 1.086 & 51986 & 1.128 & 135 & 52096 & 1.159 & 25 \\
\hline 1 & 52155.76 & 1.613 & 51890 & 1.448 & 266 & 51916 & 1.023 & 240 \\
\hline 4 & 52492.98 & 1.101 & 52595 & 1.076 & -102 & 52371 & 1.063 & 122 \\
\hline 1 & 52824.49 & 0.907 & 52935 & 0.982 & -111 & 52923 & 0.955 & -99 \\
\hline 6 & 52846.28 & 1.180 & 52977 & 1.154 & -131 & 52838 & 1.158 & 8 \\
\hline 4 & 52897.80 & 1.375 & 52983 & 1.191 & -85 & 52960 & 1.285 & -62 \\
\hline 3 & & & 52953 & 1.371 & & 52949 & 1.372 & \\
\hline 2 & 53010.95 & 1.464 & 53016 & 1.544 & -5 & 52939 & 1.609 & 72 \\
\hline 5 & 53234.65 & 1.146 & 53343 & 1.170 & -108 & 53271 & 1.170 & -36 \\
\hline 4 & 53343.56 & 1.071 & 53351 & 1.318 & -7 & 53447 & 1.257 & -103 \\
\hline 3 & 53465.79 & 1.216 & 53285 & 1.221 & 181 & 53380 & 1.165 & 86 \\
\hline 2 & 53644.83 & 1.352 & 53652 & 1.301 & -7 & 53678 & 1.267 & -33 \\
\hline 1 & 53746.80 & 1.234 & 53802 & 1.199 & -55 & 53488 & 1.214 & 259 \\
\hline 5 & 54048.81 & 1.260 & 54062 & 1.204 & -13 & 54073 & 1.208 & -24 \\
\hline 4 & 54206.69 & 1.157 & 54339 & 1.136 & -132 & 54245 & 1.102 & -38 \\
\hline 2 & 54533.78 & 1.016 & 54524 & 0.939 & 10 & 54874 & 1.077 & -340 \\
\hline 3 & 54648.73 & 0.943 & 54937 & 0.940 & -288 & 54999 & 1.280 & -350 \\
\hline 3 & 55128.38 & 1.260 & 55091 & 1.339 & 37 & 55216 & 1.055 & -88 \\
\hline 0 & 55295.66 & & 55433 & & -137 & 55168 & & -128 \\
\hline 4 & 55381.25 & 1.020 & 55607 & 1.047 & -226 & 55819 & 1.337 & -438 \\
\hline 5 & 55505.08 & 1.054 & 55560 & 1.070 & -55 & 55623 & 1.041 & -118 \\
\hline 1 & 55528.22 & 1.464 & 55312 & 1.431 & 216 & 55451 & 1.380 & 77 \\
\hline 3 & 55543.11 & 1.430 & 55558 & 1.340 & -15 & 55557 & 1.313 & -14 \\
\hline 2 & 55551.60 & 1.031 & 55532 & 1.359 & 20 & 55651 & 1.408 & -99 \\
\hline 2 & 55859.32 & 1.343 & 55878 & 1.234 & -19 & 55949 & 1.057 & -90 \\
\hline 1 & 55878.70 & 1.425 & 55973 & 1.388 & -94 & 56019 & 1.393 & -140 \\
\hline 2 & 56018.76 & 1.002 & 56180 & 0.852 & -161 & 56293 & 0.904 & -274 \\
\hline 4 & 56142.53 & 1.270 & 56057 & 1.357 & 86 & 55923 & 1.144 & 220 \\
\hline 3 & 56450.95 & 1.195 & 56452 & 1.238 & -1 & 56492 & 1.272 & -41 \\
\hline 5 & 56521.78 & 0.980 & 56612 & 0.990 & -90 & 56734 & 1.006 & -212 \\
\hline 6 & 56662.76 & & 56529 & 1.099 & 134 & 56684 & 1.096 & -21 \\
\hline 4 & 56753.05 & 1.197 & 56639 & 0.996 & 114 & 56481 & 0.895 & 272 \\
\hline 0 & & & 56640 & & & 56882 & & \\
\hline 2 & & & 56843 & 1.366 & & 56588 & 1.254 & \\
\hline 4 & 56987.88 & 1.118 & 56863 & 1.259 & 125 & 56790 & 1.254 & 198 \\
\hline 3 & 57060.81 & 1.390 & 57158 & 1.322 & -97 & 57109 & 1.241 & -48 \\
\hline 1 & 57195.81 & 1.726 & 57187 & 1.745 & 9 & 57192 & 1.773 & 4 \\
\hline
\end{tabular}


Table 3. continued.

\begin{tabular}{|c|c|c|c|c|c|c|c|c|}
\hline$J$ & $E(\mathrm{EXP})^{a}$ & $g(\mathrm{EXP})^{a}$ & $E(\mathrm{CALC})^{b}$ & $g(\mathrm{CALC})^{b}$ & $\Delta E^{b}$ & $E(\mathrm{CALC})^{c}$ & $g(\mathrm{CALC})^{c}$ & $\Delta E^{c}$ \\
\hline 2 & 57791.58 & 1.050 & 57945 & 1.010 & -153 & 57960 & 1.189 & -168 \\
\hline 4 & 58040.89 & 1.045 & 57892 & 1.054 & 149 & 57953 & 1.065 & 88 \\
\hline 1 & 58069.18 & 1.147 & 57976 & 1.086 & 93 & 57952 & 1.083 & 117 \\
\hline 3 & 58108.52 & 1.145 & 57809 & 1.128 & 300 & 57957 & 1.103 & 152 \\
\hline 6 & 58125.90 & 1.075 & 58070 & 1.087 & 56 & 58019 & 1.090 & 107 \\
\hline 2 & 58274.85 & 1.641 & 58496 & 1.709 & -221 & 58299 & 1.521 & -24 \\
\hline 5 & 58572.56 & & 58574 & 1.124 & -1 & 58575 & 1.107 & -2 \\
\hline 3 & & & 59582 & 0.949 & & 59616 & 1.000 & \\
\hline 2 & 59351.96 & 1.160 & 59624 & 1.164 & -272 & 59877 & 1.202 & -525 \\
\hline 5 & 59692.16 & & 59747 & 1.091 & -55 & 59406 & 1.105 & 286 \\
\hline 4 & 59835.92 & 1.055 & 59729 & 1.052 & 107 & 59969 & 1.023 & -133 \\
\hline 7 & 59958.59 & 1.140 & 59773 & 1.143 & 186 & 59826 & 1.143 & 132 \\
\hline 3 & 60241.07 & 1.176 & 60054 & 1.244 & 187 & 59844 & 1.243 & 397 \\
\hline 3 & 60325.15 & 1.151 & 60219 & 1.134 & 106 & 60198 & 1.104 & 127 \\
\hline 1 & 60479.60 & 0.662 & 60577 & 0.702 & -97 & 60531 & 0.679 & -51 \\
\hline 2 & 60870.30 & 1.130 & 60881 & 1.100 & -11 & 61277 & 1.049 & -407 \\
\hline 4 & 61105.69 & 1.007 & 60999 & 1.042 & 107 & 60911 & 1.092 & 195 \\
\hline 6 & 61588.16 & & 61700 & 1.092 & -112 & 61682 & 1.113 & -94 \\
\hline 3 & 61694.17 & 0.937 & 61532 & 0.924 & 162 & 61812 & 0.925 & -118 \\
\hline 4 & 62115.97 & 1.070 & 62279 & 1.054 & -163 & 62346 & 1.057 & -230 \\
\hline 5 & 62204.91 & & 62223 & 1.066 & -18 & 62362 & 1.086 & -157 \\
\hline 1 & 62296.25 & 0.600 & 62095 & 0.637 & 201 & 62237 & 0.645 & 59 \\
\hline 2 & 62317.92 & 0.986 & 62318 & 1.055 & 0 & 61770 & 1.032 & 548 \\
\hline 3 & & & 62639 & 1.158 & & 62425 & 1.179 & \\
\hline 1 & 62854.50 & 0.908 & 63048 & 0.938 & -194 & 63057 & 0.886 & -203 \\
\hline 2 & 63060.46 & 1.106 & 63093 & 0.984 & -33 & 62907 & 1.094 & 153 \\
\hline 2 & & & 63369 & 0.862 & & 63606 & 0.831 & \\
\hline 3 & & & 63550 & 1.014 & & 63175 & 1.003 & \\
\hline 0 & & & 63763 & & & 63799 & & \\
\hline 5 & 63703.35 & 0.977 & 63882 & 1.127 & -179 & 64009 & 1.117 & -306 \\
\hline 4 & 63820.47 & 1.010 & 63807 & 1.110 & 13 & 63554 & 1.109 & 266 \\
\hline 6 & 64028.85 & 1.105 & 63895 & 1.132 & 134 & 63438 & 1.105 & 591 \\
\hline 2 & & & 64007 & 0.846 & & 65021 & 0.796 & \\
\hline 3 & 64121.16 & 1.180 & 64279 & 1.145 & -158 & 64056 & 1.147 & 65 \\
\hline 1 & & & 64341 & 0.883 & & 64798 & 1.065 & \\
\hline 3 & 64653.35 & 1.079 & 64906 & 1.063 & -253 & 64898 & 1.033 & -245 \\
\hline
\end{tabular}

${ }^{a}$ From Wyart (1977) and Wyart \& Blaise $(1990) ;{ }^{b}$ this work: calculation $[\mathrm{HFR}+\mathrm{CP}(\mathrm{A})] ;{ }^{c}$ this work: calculation $[\mathrm{HFR}+\mathrm{CP}(\mathrm{B})]$.

Table 4. Calculated branching fractions (BF) and normalized transition probabilities $\left(A\right.$, in $\left.\mathrm{s}^{-1}\right)$ for transitions originating in selected odd-parity levels of Ta II $\left(E<44000 \mathrm{~cm}^{-1}\right)$. Contributions above $1 \%$ only are quoted.

\begin{tabular}{|c|c|c|c|c|c|c|}
\hline$E_{\text {upp }}\left(\mathrm{cm}^{-1}\right)$ & $J$ & $\tau(\mathrm{ns})^{c}$ & $E_{\text {low }}\left(\mathrm{cm}^{-1}\right)$ & $J$ & $B F^{a}$ & $A_{\text {NORM }}\left(\mathrm{s}^{-1}\right)^{b}$ \\
\hline \multirow{9}{*}{33706.464} & 1 & 22.8 & 0.000 & 1 & 0.3296 & $1.446(7)$ \\
\hline & & & 3180.142 & 2 & 0.2862 & $1.255(7)$ \\
\hline & & & 4124.880 & 0 & 0.1551 & $6.803(6)$ \\
\hline & & & 5330.820 & 1 & 0.0600 & $2.632(6)$ \\
\hline & & & 5657.949 & 2 & 0.0404 & $1.772(6)$ \\
\hline & & & 9690.489 & 2 & 0.0632 & $2.772(6)$ \\
\hline & & & 11875.518 & 2 & 0.0140 & $6.140(5)$ \\
\hline & & & 12600.870 & 0 & 0.0179 & $7.851(5)$ \\
\hline & & & 13475.400 & 1 & 0.0128 & $5.614(5)$ \\
\hline \multirow[t]{6}{*}{36177.194} & 2 & 13.9 & 0.000 & 1 & 0.2051 & $1.476(7)$ \\
\hline & & & 1031.418 & 2 & 0.5358 & $3.855(7)$ \\
\hline & & & 5330.820 & 1 & 0.0757 & $5.446(6)$ \\
\hline & & & 5657.949 & 2 & 0.0796 & $5.727(6)$ \\
\hline & & & 6831.441 & 3 & 0.0432 & $3.108(6)$ \\
\hline & & & 9690.489 & 2 & 0.0186 & $1.338(6)$ \\
\hline \multirow[t]{7}{*}{36763.759} & 3 & 15.6 & 1031.418 & 2 & 0.6030 & $3.865(7)$ \\
\hline & & & 2642.306 & 3 & 0.0112 & $7.179(5)$ \\
\hline & & & 3180.142 & 2 & 0.0575 & $3.686(6)$ \\
\hline & & & 5657.949 & 2 & 0.1233 & $7.904(6)$ \\
\hline & & & 6831.441 & 3 & 0.1118 & $7.167(6)$ \\
\hline & & & 9746.375 & 4 & 0.0151 & $9.679(5)$ \\
\hline & & & 14494.873 & 2 & 0.0110 & $7.051(5)$ \\
\hline
\end{tabular}


Table 4. continued.

\begin{tabular}{|c|c|c|c|c|c|c|}
\hline$E_{\text {upp }}\left(\mathrm{cm}^{-1}\right)$ & $J$ & $\tau(\mathrm{ns})^{c}$ & $E_{\text {low }}\left(\mathrm{cm}^{-1}\right)$ & $J$ & $B F^{a}$ & $A_{\mathrm{NORM}}\left(\mathrm{s}^{-1}\right)^{b}$ \\
\hline \multirow[t]{9}{*}{36987.724} & 1 & 12.2 & 0.000 & 1 & 0.1903 & $1.560(7)$ \\
\hline & & & 1031.418 & 2 & 0.1017 & $8.336(6)$ \\
\hline & & & 4124.880 & 0 & 0.4584 & $3.757(7)$ \\
\hline & & & 5330.820 & 1 & 0.0508 & $4.164(6)$ \\
\hline & & & 5657.949 & 2 & 0.0238 & $1.951(6)$ \\
\hline & & & 9690.489 & 2 & 0.0560 & $4.590(6)$ \\
\hline & & & 11875.518 & 2 & 0.0442 & $3.623(6)$ \\
\hline & & & 12600.870 & 0 & 0.0291 & $2.385(6)$ \\
\hline & & & 13560.282 & 2 & 0.0167 & $1.369(6)$ \\
\hline \multirow[t]{4}{*}{37230.738} & 2 & 5.4 & 0.000 & 1 & 0.6627 & $1.227(8)$ \\
\hline & & & 1031.418 & 2 & 0.2367 & $4.383(7)$ \\
\hline & & & 2642.306 & 3 & 0.0159 & $2.945(6)$ \\
\hline & & & 5330.820 & 1 & 0.0445 & $8.241(6)$ \\
\hline \multirow[t]{10}{*}{38515.698} & 2 & 14.4 & 0.000 & 1 & 0.3452 & $2.397(7)$ \\
\hline & & & 2642.306 & 3 & 0.0372 & $2.583(6)$ \\
\hline & & & 3180.142 & 2 & 0.0111 & $7.708(5)$ \\
\hline & & & 5330.820 & 1 & 0.2906 & $2.018(7)$ \\
\hline & & & 5657.949 & 2 & 0.0304 & $2.111(6)$ \\
\hline & & & 6831.441 & 3 & 0.1440 & $1.000(7)$ \\
\hline & & & 10713.300 & 1 & 0.0177 & $1.229(6)$ \\
\hline & & & 11767.262 & 3 & 0.0166 & $1.153(6)$ \\
\hline & & & 13475.400 & 1 & 0.0222 & $1.542(6)$ \\
\hline & & & 14581.064 & 3 & 0.0403 & $2.799(6)$ \\
\hline \multirow[t]{6}{*}{38535.375} & 1 & 6.9 & 0.000 & 1 & 0.3250 & $4.710(7)$ \\
\hline & & & 1031.418 & 2 & 0.2994 & $4.339(7)$ \\
\hline & & & 3180.142 & 2 & 0.0748 & $1.084(6)$ \\
\hline & & & 4124.880 & 0 & 0.1560 & $2.261(7)$ \\
\hline & & & 5657.949 & 2 & 0.0937 & $1.358(7)$ \\
\hline & & & 9690.489 & 2 & 0.0231 & $3.348(6)$ \\
\hline \multirow[t]{3}{*}{38962.375} & 3 & 6.2 & 1031.418 & 2 & 0.5868 & $9.465(7)$ \\
\hline & & & 2642.306 & 3 & 0.3656 & $5.897(7)$ \\
\hline & & & 9690.489 & 2 & 0.0183 & $2.952(6)$ \\
\hline \multirow[t]{6}{*}{39743.630} & 4 & 12.6 & 2642.306 & 3 & 0.6900 & $5.476(7)$ \\
\hline & & & 6831.441 & 3 & 0.0833 & $6.611(6)$ \\
\hline & & & 9746.375 & 4 & 0.0586 & $4.651(6)$ \\
\hline & & & 11767.262 & 3 & 0.0875 & $6.944(6)$ \\
\hline & & & 14581.064 & 3 & 0.0258 & $2.048(6)$ \\
\hline & & & 15726.107 & 3 & 0.0222 & $1.762(6)$ \\
\hline \multirow[t]{12}{*}{41145.119} & 2 & 4.8 & 0.000 & 1 & 0.1220 & $2.542(7)$ \\
\hline & & & 1031.418 & 2 & 0.1386 & $2.887(7)$ \\
\hline & & & 2642.306 & 3 & 0.2217 & $4.619(7)$ \\
\hline & & & 5330.820 & 1 & 0.1512 & $3.150(7)$ \\
\hline & & & 5657.949 & 2 & 0.1406 & $2.929(7)$ \\
\hline & & & 6831.441 & 3 & 0.0242 & $5.042(6)$ \\
\hline & & & 9690.489 & 2 & 0.0138 & $2.875(6)$ \\
\hline & & & 10713.300 & 1 & 0.0375 & $7.812(6)$ \\
\hline & & & 11767.262 & 3 & 0.0505 & $1.052(7)$ \\
\hline & & & 13475.400 & 1 & 0.0198 & $4.125(6)$ \\
\hline & & & 13560.282 & 2 & 0.0192 & $4.000(6)$ \\
\hline & & & 14581.064 & 3 & 0.0358 & $7.458(6)$ \\
\hline \multirow[t]{7}{*}{41775.291} & 4 & 6.4 & 2642.306 & 3 & 0.3446 & $5.384(7)$ \\
\hline & & & 4415.764 & 4 & 0.5124 & $8.006(7)$ \\
\hline & & & 6831.441 & 3 & 0.0445 & $6.953(6)$ \\
\hline & & & 9746.375 & 4 & 0.0104 & $1.625(6)$ \\
\hline & & & 11767.262 & 3 & 0.0131 & $2.047(6)$ \\
\hline & & & 12435.850 & 3 & 0.0301 & $4.703(6)$ \\
\hline & & & 12705.402 & 4 & 0.0216 & $3.375(6)$ \\
\hline \multirow[t]{9}{*}{42959.625} & 3 & 6.0 & 1031.418 & 2 & 0.0652 & $1.087(7)$ \\
\hline & & & 2642.306 & 3 & 0.0703 & $1.172(7)$ \\
\hline & & & 3180.142 & 2 & 0.0145 & $2.417(6)$ \\
\hline & & & 4415.764 & 4 & 0.3202 & $5.337(7)$ \\
\hline & & & 5657.949 & 2 & 0.3361 & $5.602(7)$ \\
\hline & & & 9690.489 & 2 & 0.0262 & $4.367(6)$ \\
\hline & & & 9746.375 & 4 & 0.0277 & $4.617(6)$ \\
\hline & & & 11767.262 & 3 & 0.0818 & $1.363(7)$ \\
\hline & & & 12966.020 & 4 & 0.0197 & $3.283(6)$ \\
\hline
\end{tabular}


Table 4. continued.

\begin{tabular}{|c|c|c|c|c|c|c|}
\hline$E_{\text {upp }}\left(\mathrm{cm}^{-1}\right)$ & $J$ & $\tau(\mathrm{ns})^{c}$ & $E_{\text {low }}\left(\mathrm{cm}^{-1}\right)$ & $J$ & $B F^{a}$ & $A_{\mathrm{NORM}}\left(\mathrm{s}^{-1}\right)^{b}$ \\
\hline 43064.948 & 2 & 9.0 & $\begin{array}{r}1031.418 \\
2642.306 \\
3180.142 \\
5657.949 \\
6831.441 \\
9690.489 \\
10713.300 \\
11875.518 \\
13475.400 \\
13560.282 \\
17375.110 \\
23294.700\end{array}$ & $\begin{array}{l}2 \\
3 \\
2 \\
2 \\
3 \\
2 \\
1 \\
2 \\
1 \\
2 \\
1 \\
2\end{array}$ & $\begin{array}{l}0.1537 \\
0.1766 \\
0.0246 \\
0.2845 \\
0.1446 \\
0.0114 \\
0.0277 \\
0.0198 \\
0.0187 \\
0.0679 \\
0.0144 \\
0.0113\end{array}$ & $\begin{array}{l}1.708(7) \\
1.962(7) \\
2.733(6) \\
3.161(7) \\
1.607(7) \\
1.267(6) \\
3.078(6) \\
2.200(6) \\
2.078(6) \\
7.544(6) \\
1.600(6) \\
1.444(6)\end{array}$ \\
\hline
\end{tabular}

${ }^{a}$ This work: calculation HFR + CP(A) (see the text); ${ }^{b}$ normalized transition probabilities (in s ${ }^{-1}$ ). $a(b)$ is written for $a 10^{b} ;{ }^{c}$ lifetime value adopted for the normalization. When several experimental results are available, a mean value has been calculated (see the text).

Table 5. Radiative lifetimes for odd-parity levels of Ta II (in ns) $\left(E<44000 \mathrm{~cm}^{-1}\right)$.

\begin{tabular}{lllrrr}
\hline \hline$E\left(\mathrm{~cm}^{-1}\right)$ & $J$ & $\begin{array}{l}\text { Exp. } \\
\text { Previous }\end{array}$ & $\begin{array}{r}\text { Exp. } \\
\text { This work }\end{array}$ & $\begin{array}{r}\text { HFR + CP(A) } \\
\text { This work }\end{array}$ & $\begin{array}{r}\text { HFR + CP(B) } \\
\text { This work }\end{array}$ \\
\hline 33706.464 & 1 & & $22.8(1.0)$ & 23.1 & 29.8 \\
36177.194 & 2 & $13.9(9)^{c}$ & & 10.4 & 14.0 \\
36763.759 & 3 & $15.4(9)^{a}, 15.8(9)^{c}$ & 16.7 & 21.2 \\
36987.724 & 1 & $12.2(8)^{c}$ & 7.7 & 10.1 \\
37230.738 & 2 & $5.5(3)^{a}, 5.2(5)^{b}, 5.6(3)^{c}$ & & 5.2 & 6.2 \\
38515.698 & 2 & $13.2(9)^{a}, 14.7(15)^{b}, 15.3(8)^{c}$ & & 13.3 & 16.5 \\
38535.375 & 1 & $6.9(4)^{a}, 6.6(6)^{b}, 7.1(3)^{c}$ & & 7.0 & 8.7 \\
38962.375 & 3 & $6.2(4)^{c}$ & & 3.6 & 4.5 \\
39295.954 & 3 & $15.4(8)^{a}, 13.6(14)^{b}, 15.8(8)^{c}$ & & 13.4 & 60.6 \\
39743.630 & 4 & $12.6(9)^{c}$ & & 5.1 & 15.1 \\
41145.119 & 2 & $4.8(4)^{a}, 4.8(5)^{b}$ & & 4.9 & 5.9 \\
41775.291 & 4 & $6.6(5)^{c}, 6.2(6)^{e}$ & & 6.3 & 6.7 \\
42959.625 & 3 & & $6.0(4)$ & 6.6 & 5.8 \\
43064.948 & 2 & & $9.0(5)$ & & 8.8 \\
\hline
\end{tabular}

${ }^{a}$ Kwiatkowski et al. (1984); ${ }^{b}$ Bergström et al. (1986); ${ }^{c}$ Schade \& Helbig (1986); ${ }^{d}$ Langhans et al. (1995); ${ }^{e}$ Henderson et al. (1999).

with a risetime of $0.2 \mathrm{~ns}$ and a fall time of $0.6 \mathrm{~ns}$. The signal was recorded by a transient digitizer with a time resolution of $0.5 \mathrm{~ns}$.

In the measurements, the laser pulse and the fluorescence signal were recorded alternatively with the same detection system.

The new experimental lifetime values are reported in Table 5 for the three levels considered at 33706, 42960, and $43065 \mathrm{~cm}^{-1}$. These 3 lifetimes are averages for at least ten recordings. The error bars take into account the statistical uncertainties from the fitting as well as variations between the different recordings. There are no other experimental lifetimes available for comparison.

\subsection{Discussion}

The calculated lifetimes are given in Table 5 for the low-lying levels $\left(E<44000 \mathrm{~cm}^{-1}\right)$ of the odd configurations for which they are compared with the previous experimental results, and also with the 3 new lifetimes obtained in the present work.

It is obvious that the theoretical values for the level at $39296 \mathrm{~cm}^{-1}$, which disagree with the two experimental values available, are unreliable, the reason being cancellation effects affecting some depopulation channels of this level.
For two levels measured in the present work, the model $\mathrm{HFR}+\mathrm{CP}(\mathrm{B})$ is in better agreement with the experimental results than the model HFR $+\mathrm{CP}(\mathrm{A})$, while the opposite is true for the third level. On the one hand, the model A is in good agreement with the experimental results of both Kwiatkowski et al. (1984) and Bergström et al. (1986). For some levels, on the other hand, the model $\mathrm{B}$ agrees quite well with the experimental results of Schade \& Helbig (1986). On the basis of this comparison, it is not obvious to decide which model provides the best description of the data. However, the BFs obtained in the two calculations agree quite well (to within a few percent of each other particularly for the strongest transitions).

The transition probabilities $(A)$ for the strongest transitions depopulating the levels of Table 5 are presented in Table 4 . They were obtained by combining the experimental lifetimes (either measured in the present work or taken from previous analyses; when several values were available, the mean value was adopted; see Col. 3 of Table 4) with theoretical BFs (which were calculated in the present work with the model A).

Table 4 has been limited to the lines with depopulation channels for which $g A>10^{8} \mathrm{~s}^{-1}$. Additional results, for the weaker transitions, will be available in the database DESIRE ${ }^{1}$.

\footnotetext{
1 This database is available at the URL address: http://w3 . umh . ac. be/ astro/desire.shtml
} 


\section{Conclusions}

On the basis of the laser lifetime measurements and HFR calculations performed in the present work, the following conclusions are valid:

1. New BFs and $A$-values have been obtained for a large set of Ta II transitions of astrophysical interest.

2. The accuracy of the results has been assessed by comparing HFR lifetime values with experimental results obtained using the time-resolved, laser-induced, fluorescence technique. The agreement observed between the theoretical results and experimental measurements is good.

3. The new results are expected to be accurate to within a few percent at least for the most intense transitions. Large inaccuracies are possible for the weaker lines, particularly when cancellation effects are present in the calculation of the line strengths. These new results are expected to be useful to astrophysicists investigating some stellar spectra.

4. The present work calls for additional experimental investigations of branching fractions or transition probabilities in this ion.

\footnotetext{
Acknowledgements. This work was financially supported by the Integrated Initiative of Infrastructure Project LASERLAB-EUROPE, contract RII3-CT2003-506350, the Swedish Research Council through the Linneus grant and the Belgian FRS-FNRS. E.B., P.Q. and P.P. are, respectively, Research Director, Senior Research Associate and Research Associate of the FRS-FNRS. V.F. acknowledges a fellowship from FRIA. We are grateful to Prof. S. Svanberg (Lund Laser Centre) for his enthousiastic support.
}

\section{References}

Asplund, M., Grevesse, N., \& Sauval, A. J. 2006, Nucl. Phys. A, 777, 1 Bergström, H., Lundberg, H., Persson, A., Schade, W., \& Zhao, Y. Y. 1986, Phys. Scr., 33, 513

Biémont, É. 2005, Phys. Scr. T, 119, 55

Biémont, É., \& Quinet, P. 2003, Phys. Scr. T, 105, 38

Corliss, C. H., \& Bozman, W. R. 1962, Nat. Bur. Stand. (US), Monogr. 53, Washington D.C.

Cowan, R. D. 1981, The Theory of Atomic Structure and Spectra (Berkeley: University of California Press)

Eriksson, M., Litzén, U., Wahlgren, G. M., \& Leckrone, D. S. 2002, Phys. Scr., 65,480

Fivet, V., Palmeri, P., Quinet, P., et al. 2006, Eur. Phys. J. D, 37, 29

Fivet, V., Biémont, É., Engström, L., et al. 2008, J. Phys. B: At. Mol. Opt. Phys., 41, 015702

Fraga, S., Karwowski, J., \& Saxena, K. M. S. 1976, Handbook of Atomic Data (Amsterdam: Elsevier)

Henderson, M., Irving, R. E., Matulioniene, R., et al. 1999, ApJ, 520, 805

Kiess, C. C. 1962, J.R.N.B.S., 66A, 111

Kwiatkowski, M., Naumann, F., Werner, K., \& Zimmermann, P. 1984, Phys. Lett. A, 103, 49

Langhans, G., Schade, W., \& Helbig, V. 1995, Z. Phys. D, 34, 151

Moore, C. E. 1958, Atomic Energy Levels, Vol. III, NBS Circ., 467

Quinet, P., Palmeri, P., Biémont, É., et al. 1999, MNRAS, 307, 934

Schade, W., \& Helbig, V. 1986, Phys. Lett. A, 115, 39

Wyart, J.-F., \& Blaise, J. 1990, Phys. Scr., 42, 209

Wyart, J.-F. 1977, Optica Pura y Aplic., 10, 177

Xu, H. L., Jiang, Z. K., Zhang, Z. G., et al. 2003, J. Phys. B: At. Mol. Opt. Phys., 36,1771

Xu, H. L., Persson, A., Svanberg, S., et al. 2004, Phys. Rev. A, 70, 042508 